\title{
Fuzzy intelligent control method for improving flight attitude stability of plant protection quadrotor UAV
}

\author{
Zhihui $\mathrm{He}^{1,2}$, Wanlin $\mathrm{Gao}^{1,2^{*}}$, Xiongkui He ${ }^{1,3^{*}}$, Minjuan Wang ${ }^{1,2}$, Yunling $\mathrm{Liu}^{1,2}$, \\ Yue Song ${ }^{1,2}$, Zewu $\mathrm{An}^{4}$ \\ (1. College of Information and Electrical Engineering, China Agricultural University, Beijing100083, China; \\ 2. Key Laboratory of Agricultural Informationization Standardization, Ministry of Agriculture and Rural Affairs, Beijing 100083, China; \\ 3. Centre for Chemicals Application Technology, College of Science, China Agricultural University, Beijing 100083, China; \\ 4. School of Mechanical Engineer \& Automation, Beihang University, Beijing 100191, China)
}

\begin{abstract}
At present, the attitude control method of plant protection UAV is the classical PID control, but there are some imperfections in the PID control, such as the contradiction between speediness and overshoot, the weak anti-jamming ability and adaptability. The physical parameters of plant protection UAV are time-varying, and the airflow also interferes with it. The control ability of classical PID is limited, and its control parameters are fixed, and its anti-jamming ability and adaptability are not strong. Therefore, a fuzzy adaptive PID controller is proposed in this paper. Fuzzy logic control is used to optimize the control parameters of PID in order to improve the dynamic and static performance and adaptability of attitude control of plant protection UAV. In the process of research, the mathematical model of UAV is established firstly, then the fuzzy adaptive PID is designed, and then the simulation is carried out in Simulink. The simulation results show that the fuzzy adaptive PID controller has better dynamic and static control performance and adaptability than the traditional PID controller. Therefore, the proposed control method has excellent application value in the attitude of plant protection UAV.
\end{abstract}

Keywords: quadrotor UAV, attitude control, plant protect, fuzzy adaptive PID, Simulink

DOI: $10.25165 /$ j.ijabe.20191206.5108

Citation: He Z H, Gao W L, He X K, Wang M J, Liu Y L, Song Y, et al. Fuzzy intelligent control method for improving flight attitude stability of plant protection quadrotor UAV. Int J Agric \& Biol Eng, 2019; 12(6): 110-115.

\section{Introduction}

Agricultural plant protection UAV has increasing broad application prospects in modern agricultural production ${ }^{[1]}$. On the one hand, the complex working environment increases the test of the stability of the drone. On the other hand, agricultural plant protection drones operate in low-altitude, low-speed environments, and the flight is greatly affected by the environment, which makes it difficult to control the operation of agricultural drones. In addition, the sensors on the agricultural plant protection drone are limited by cost, volume and weight, and the accuracy will be affected, which will affect the flight control of the plant protection drone. In this case, it is especially important to develop an

Received date: 2019-04-24 Accepted date: 2019-09-24

Biographies: Zhihui He, PhD candidate, research interest: design and simulation of attitude control system for UAV, Email: hezh213@126.com; Minjuan Wang, PhD, research interest: artificial intelligence system, Email: minjuan@cau.edu.cn; Yunlin Liu, PhD, Associate Professor, research interest: application of agricultural UAV, Email: lyunling@163.com; Yue Song, PhD candidate, research interest: speech recognition, Email: songyue597@163.com; Zewu An, Master candidate, research interest: artificial intelligence system, Email: zewuan@buaa.edu.cn.

*Corresponding author: Wanlin Gao, $\mathrm{PhD}$, Professor, research interest: agricultural informationization and application specific chip. College of Information and Electrical Engineering, China Agricultural University, No.17, Qinghua East Road, Haidian District, Beijing 100083, China. Tel: +86-1062738535, Email: wanlin_cau@163.com; Xiongkui He, PhD, Professor, research focuses on pesticide application technology. Centre for Chemicals Application Technology (CCAT), China Agricultural University, No.2, Yuanmingyuan West Road, Haidian District, Beijing 100193, China. Tel: +86-10-62731446, Email: xiongkui@cau.edu.cn. excellent attitude control method

At present, domestic plant protection drones generally use traditional PID control algorithms. Aiming at the small-area planting area and precision agriculture development demand in southern China, Huang ${ }^{[2]}$ designed a small quadrotor plant protection drone using the classical PID control method. In response to the operational characteristics and application fields of agricultural drones, Liu et al. ${ }^{[3]}$ designed the longitudinal attitude control system for agricultural drones using classical PID control methods. In view of the limited performance of PID control, Liu hopes to apply more efficient control methods to flight control of agricultural drones. In order to solve the problem that plant protection drones cannot achieve obstacle avoidance, $\mathrm{Qiu}^{[4]}$ developed a flight control system suitable for agricultural plant protection drones based on classical PID control.

However, there are some imperfections in the structure of the classic PID controller ${ }^{[5,6]}$. For example, there are contradictions in the rapidity of control and overshoot. The effect of suppressing time-varying disturbances is not significant, and the adaptability of classical PID control is not strong. In this case, the use of the classic PID control method will limit the attitude control performance of the plant protection drone. The reason is that the three control parameters (proportional gain, integral gain and differential gain) of the PID control cannot be adjusted at any time with the change of the environment. Therefore, in order to improve the attitude control performance of plant protection drones, this paper proposes a fuzzy adaptive PID control method. The fuzzy control combines the traditional expert system based on the rules, fuzzy set theory and the achievements of cybernetics ${ }^{[7]}$. Combining fuzzy control with classic PID control will achieve 
better results than classic PID control ${ }^{[8,9]}$.

The purpose of this study is to introduce fuzzy logic control based on classical PID control, optimize the parameters of PID, improve the attitude control performance of plant protection UAV, and improve the adaptability of plant protection UAV. In the process of research, we first use the Newton-Euler method to establish the mathematical model of the UAV, then design a fuzzy adaptive PID control algorithm, and carry out simulation experiments in Simulink. The experimental results show that the proposed method is feasible.

\section{Dynamic modeling of quadrotor UAV}

In order to establish the mathematical model of quadrotor UAV conveniently, firstly, the flight conditions of quadrotor UAV are rationally simplified, then establishes the system reference frame, carries out force analysis and moment analysis of the quadrotor UAV on the basis of the assumptions, and finally establishes the dynamic mathematical model of the quadrotor UAV. In this paper, a quadrotor UAV with a cross structure is shown in Figure 1.

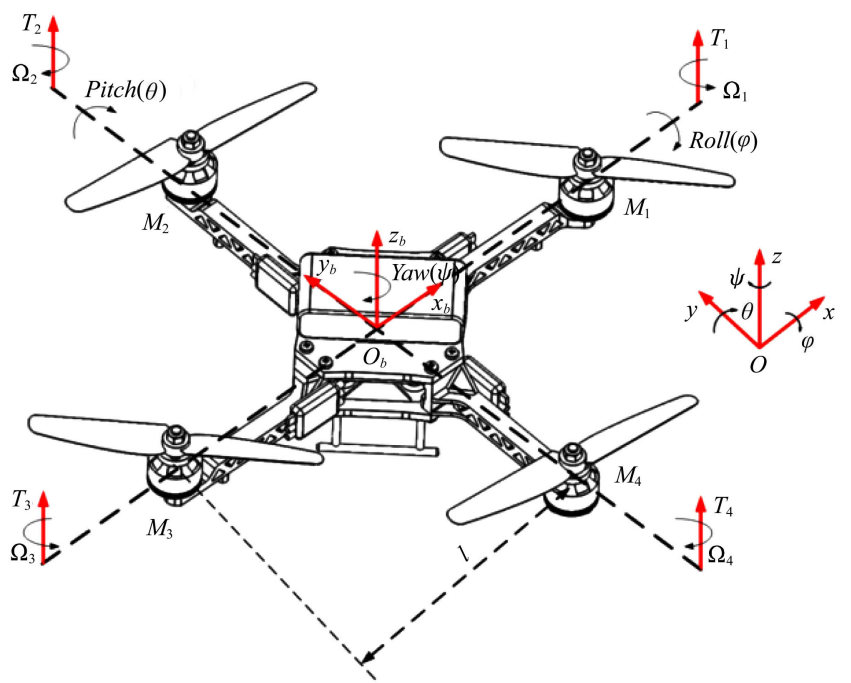

Figure 1 Cross type structure quadrotor UAV

It is difficult to mathematically model the quadrotor flight system in the actual flight process, such as the external airflow conditions and the inherent influence of the internal airframe structure. In order to facilitate modeling, the following reasonable assumptions are made for these uncertainties ${ }^{[10]}$.

1) The quadrotor is a symmetrical rigid body;

2) The origin of the inertial coordinate system is in the same position as the geometric center and centroid of the quadrotor;

3) Resistance and gravity of the quadrotor do not be affected by flight altitude and other factors;

4) Tensions in all directions are proportional to the square of the propeller speed.

An adequate dynamic system model was vital to the simulation of the control strategy. In order to get the mathematical modeling for the quadrotor, two coordinate systems were established, which were inertial coordinate system $\boldsymbol{E}(\mathrm{O} x y z)$ and body coordinate system $\boldsymbol{B}\left(\mathrm{O}_{b} x_{b} y_{b} z_{b}\right)$, shown in Figure 1. These two coordinates can be converted to each other through transition matrix $\boldsymbol{R}$. The transformation matrix $\boldsymbol{R}$ can be expressed by Euler angle ${ }^{[11]}$. Euler angles can be used to describe the quadrotor's attitude, roll $(\varphi)$, pitch $(\theta)$, and yaw $(\psi)$. In Figure 2, the Euler angles are defined as follows: Yaw angle $\psi$ is the angle between the $\mathrm{X}$-axis and the projection of $O x$ in the $O X Y$ plane; Pitch angle $\theta$ is the angle between the $Z$-axis and the projection of $O z$ in the $O X Z$ plane; Roll angle $\varphi$ is the angle between the $\mathrm{Y}$-axis and the projection of $O y$ in the $O Y Z$ plane.
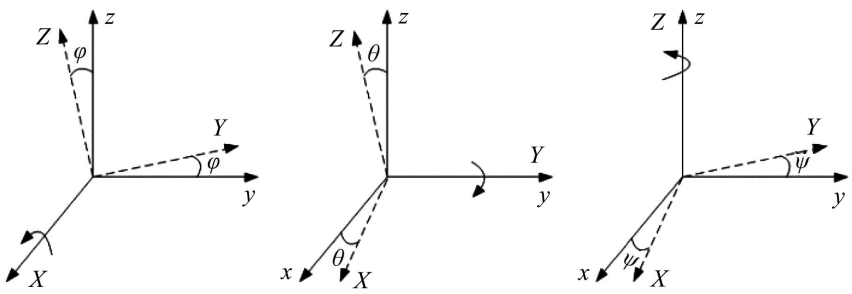

Figure 2 Diagram of Euler angle

Get the transition matrix $\boldsymbol{R}$ from the body coordinate system $\boldsymbol{B}\left(\mathrm{O}_{b} x_{b} y_{b} z_{b}\right)$ to the inertial coordinate system $\boldsymbol{E}(O x y z)$.

\section{$\boldsymbol{R}=\boldsymbol{R} x \cdot \boldsymbol{R} y \cdot \boldsymbol{R} z$}

$$
=\left[\begin{array}{ccc}
c \psi c \theta & -s \psi c \varphi+c \psi s \theta s \varphi & s \psi s \varphi+c \psi s \theta s \varphi \\
s \psi c \theta & c \psi c \varphi+s \psi s \theta s \varphi & -c \psi s \varphi+s \psi s \theta s \varphi \\
-s \theta & c \theta s \varphi & c \theta c \varphi
\end{array}\right]
$$

where, $s$ and $c$ denote sine and cosine functions respectively, i.e., $c \psi \mathrm{c} \theta=\cos \psi \cos \theta, s \psi c \varphi=\sin \psi \cos \varphi$.

Newton's second law and aircraft dynamics equation ${ }^{[12,13]}$ can be expressed as vectors, respectively, as follows:

$$
\begin{gathered}
F=m \frac{d V}{d t} \\
M=m \frac{d H}{d t}
\end{gathered}
$$

where, $F$ is the external force acting on the quadrotor aircraft; $m$ is the mass of the quadrotor aircraft; $V$ is the speed of the aircraft; $M$ is the sum of the momentum acting on the quadrotor aircraft; $H$ is the absolute momentum of the quadrotor aircraft relative to the inertial coordinate system. Gravity $G$, lift $T_{i}$ and reverse torque $D_{i}$ of the single rotor are expressed as follows.

$$
G=m g, T_{i}=\rho C_{t} \omega_{i}^{2} / 2=k_{t} \omega_{i}^{2}, D_{i}=\rho C_{d} \omega_{i}^{2} / 2=k_{d} \omega_{i}^{2}
$$

where, $k_{t}$ and $k_{d}$ are force and moment constants and $\omega_{i}$ is the propeller's rotation speed.

According to the force analysis, Newton's second law and aircraft dynamics equation can be obtained linear motion equation, which is expressed as follows:

$$
\left\{\begin{aligned}
\ddot{x} & =\left(F_{x}-K_{1} \dot{x}\right) / m \\
& =\left(k_{t} \sum_{i=1}^{4} \omega_{i}^{2}(\cos \psi \sin \theta \cos \varphi+\sin \psi \sin \varphi)-K_{1} \dot{x}\right) / m \\
\ddot{y} & =\left(F_{y}-K_{2} \dot{y}\right) / m \\
& =\left(k_{t} \sum_{i=1}^{4} \omega_{i}^{2}(\sin \psi \sin \theta \cos \varphi-\cos \psi \sin \varphi)-K_{2} \dot{y}\right) / m \\
\ddot{z} & =\left(F_{z}-K_{3} \dot{z}-m g\right) / m \\
& =\left(k_{t} \sum_{i=1}^{4} \omega_{i}^{2}\left(\cos \theta \cos \varphi-K_{3} \dot{z}\right)\right) / m-g
\end{aligned}\right.
$$

where, $F_{x}, F_{y}, F_{z}$ are defined as the components of $\boldsymbol{F}$ on the three coordinate axes of the body coordinate system; $K_{1}, K_{2}, K_{3}$ are the resistance coefficients in linear motion.

According to the relationship between Euler angles and angular velocities of quadrotor aircraft, the following set of formulas can be obtained.

$$
\left[\begin{array}{l}
p \\
q \\
r
\end{array}\right]=\left[\begin{array}{c}
\dot{\varphi}-\dot{\psi} \sin \theta \\
\dot{\theta} \cos \varphi+\dot{\psi} \sin \varphi \cos \theta \\
-\dot{\theta} \sin \theta+\dot{\psi} \cos \varphi \cos \theta
\end{array}\right]
$$




$$
\left[\begin{array}{c}
\dot{\varphi} \\
\dot{\theta} \\
\dot{\psi}
\end{array}\right]=\left[\begin{array}{c}
(p \cos \theta+q \sin \varphi \sin \theta+r \cos \varphi \sin \theta) / \cos \theta \\
q \cos \varphi+r \sin \varphi \\
(q \sin \varphi+r \cos \varphi) / \cos \theta
\end{array}\right]
$$

where, $p, q$, rare components of angular velocity $\omega$ on the three coordinate axes of the body coordinate system.

As previously assumed, the mass and structure of a quadrotor aircraft are uniformly symmetrical, so its inertia matrix can be defined as diagonal matrix $I$.

$$
I=\left[\begin{array}{ccc}
I_{x} & & \\
& I_{y} & \\
& & I_{z}
\end{array}\right]
$$

According to $\boldsymbol{M}=m \frac{d H}{d t}$, we can get

$$
\left[\begin{array}{l}
M_{x} \\
M_{y} \\
M_{z}
\end{array}\right]=I\left[\begin{array}{l}
\dot{p} \\
\dot{q} \\
\dot{r}
\end{array}\right]
$$

By calculating the angular momentum, the angular motion equations of the three-axis components of $\boldsymbol{M}$ in the body coordinate system, $M_{x}, M_{y}$ and $M_{z}$, are obtained.

$$
\left[\begin{array}{l}
M_{x} \\
M_{y} \\
M_{z}
\end{array}\right]=I\left[\begin{array}{l}
\dot{p} I_{x}-\dot{r} I_{x z}+q r\left(I_{z}-I_{y}\right)-p q I_{x z} \\
\dot{q} I_{y}+p r\left(I_{x}-I_{z}\right)+\left(p^{2}-r^{2}\right) I_{x z} \\
\dot{r} I_{z}-\dot{p} I_{x z}+p q\left(I_{y}-I_{x}\right)+q r I_{x z}
\end{array}\right]
$$

According to diagonal matrix $I$, the above set of formulas can be simplified as the following one

$$
\left[\begin{array}{c}
\dot{p} \\
\dot{q} \\
\dot{r}
\end{array}\right]=\left[\begin{array}{l}
{\left[M_{x}+\left(I_{z}-I_{y}\right) q r\right] / I_{x}} \\
{\left[M_{y}+\left(I_{x}-I_{z}\right) p r\right] / I_{y}} \\
{\left[M_{z}+\left(I_{y}-I_{x}\right) p q\right] / I_{z}}
\end{array}\right]
$$

Combining the above linear motion equation with the angular motion equation, the quadrotor nonlinear motion equation is obtained.

$$
\left\{\begin{aligned}
\ddot{x} & =\left(F_{x}-K_{1} \dot{x}\right) / m \\
& =\left(k_{t} \sum_{i=1}^{4} \omega_{i}^{2}(\cos \psi \sin \theta \cos \varphi+\sin \psi \sin \varphi)-K_{1} \dot{x}\right) / m \\
\ddot{y} & =\left(F_{y}-K_{2} \dot{y}\right) / m \\
& =\left(k_{t} \sum_{i=1}^{4} \omega_{i}^{2}(\sin \psi \sin \theta \cos \varphi-\cos \psi \sin \varphi)-K_{2} \dot{y}\right) / m \\
\ddot{z} & =\left(F_{z}-K_{3} \dot{z}-m g\right) / m \\
& =\left(k_{t} \sum_{i=1}^{4} \omega_{i}^{2}\left(\cos \theta \cos \varphi-K_{3} \dot{z}\right)\right) / m-g \\
\dot{p} & =\left[M_{x}+\left(I_{z}-I_{y}\right) q r\right] / I_{x} \\
\dot{q} & =\left[M_{y}+\left(I_{x}-I_{z}\right) p r\right] / I_{y} \\
\dot{r} & =\left[M_{z}+\left(I_{y}-I_{x}\right) p q\right] / I_{z} \\
\dot{\varphi} & =(p \cos \theta+q \sin \varphi \sin \theta+r \cos \varphi \sin \theta) / \cos \theta \\
\dot{\theta} & =q \cos \varphi+r \sin \varphi \\
\dot{\psi} & =(q \sin \varphi+r \cos \varphi) / \cos \theta
\end{aligned}\right.
$$

The quadrotor is subjected to one force and three moments ${ }^{[14]}$. These force and moments represent the inputs of the four independent control inputs to quadrotor and they can be defined as vertical force input $U_{1}$, pitch moment input $U_{2}$, roll moment input $U_{3}$, yaw moment input $U_{4}$

$$
\begin{aligned}
{\left[\begin{array}{l}
U_{1} \\
U_{2} \\
U_{3} \\
U_{4}
\end{array}\right]=\left[\begin{array}{c}
T_{1}+T_{2}+T_{3}+T_{4} \\
\left(T_{4}-T_{2}\right) l \\
\left(T_{3}-T_{1}\right) l \\
D_{2}+D_{4}-D_{1}-D_{3}
\end{array}\right] } \\
=\left[\begin{array}{c}
k_{t} \sum_{i=1}^{4} \omega_{i}^{2} \\
k_{t}\left(\omega_{4}^{2}-\omega_{2}^{2}\right) l \\
k_{t}\left(\omega_{3}^{2}-\omega_{1}^{2}\right) l \\
k_{d}\left(\omega_{2}^{2}+\omega_{4}^{2}-\omega_{1}^{2}-\omega_{3}^{2}\right)
\end{array}\right]
\end{aligned}
$$

where, $T_{i}, D_{i}, k_{t}, k_{d}$ have been defined earlier and $l$ is the distance from the center of the rotor to the origin of the body coordinate system.

In the case of no wind and low-speed flight, the drag coefficients $K_{1}, K_{2}, K_{3}$ are negligible. In addition to the torque generated by actuators, the quadrotor UAV is also affected by the gyroscopic effect caused by the airframe or rotor. However, the effect of the gyroscopic effect, especially in hovering or low-speed flight, is negligible compared with that produced by actuators, which can be simplified as a small disturbance of the system, so we can get a simplified model.

$$
\left\{\begin{array}{l}
\ddot{x}=(\cos \psi \sin \theta \cos \varphi+\sin \psi \sin \varphi) U_{1} / m \\
\ddot{y}=(\sin \psi \sin \theta \cos \varphi-\cos \psi \sin \varphi) U_{1} / m \\
\ddot{z}=(\cos \theta \cos \varphi) U_{1} / m-g \\
\ddot{\varphi}=U_{2} / I_{x} \\
\ddot{\theta}=U_{3} / I_{y} \\
\ddot{\psi}=U_{4} / I_{z}
\end{array}\right.
$$

\section{Attitude control algorithms design}

In this section, two controllers for the attitude control of the quadrotor are presented, which are the traditional PID controller and the fuzzy adaptive PID controller, respectively.

As mentioned before the inputs chose like equations (11), where $U_{1}$ controls the motion along the z-axis, $U_{2}$ controls rotation along the $x$-axis (roll angle), $U_{3}$ controls the rotation along the $\mathrm{y}$-axis (pitch angle), and $U_{4}$ controls rotation along the z-axis (yaw angle). The designed controllers should set values to parameters which determine the four rotor speed parameters by equations (12).

\subsection{Traditional PID controller}

The quadrotor UAV system has only four input control variables, but there are six degrees of freedom, and the six constraints relation are existed among the six outputs, so it is difficult to control the position and attitude accurately at the same time $^{[15]}$. The nested loops control architecture was employed to control the flight position and attitude respectively, as shown in Figure 3. The PID control was used in each loop. Note that this paper only studies four channels: altitude, roll, pitch and yaw, so the $x, y$ channels in Figure 3 are dotted.

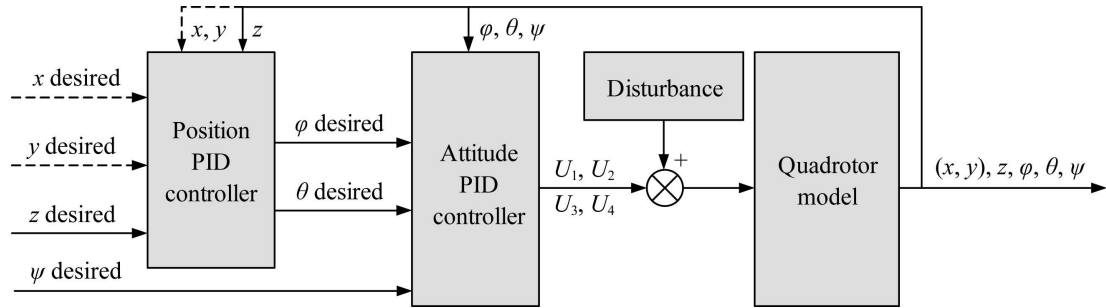

Figure 3 PID Control diagram of the quadrotor UAV system 
The equation of a PID controller has the following form:

$$
u(t)=K_{p} \cdot e(t)+K_{i} \cdot \int e(t) d t+K_{d} \cdot \frac{d e(t)}{d t}
$$

where, $K_{p}, K_{i}$ and $K_{d}$ are called the proportional, integral and differential gains, respectively; $e(t)$ is the error between the expected value and the actual value.

\subsection{Fuzzy system for tuning the PID gains}

The success of the PID controller depends on the appropriate PID gains. Tuning the PID gains is very important to optimize performance. In practice, the PID gains are usually tuned by experienced human experts based on some "rule of thumb". In this work, we first determined a set of tuning rules (fuzzy IF-THEN rules) for the PID gains, and then combine these rules into a fuzzy system that is used to adjust the PID gains on-line ${ }^{[16]}$.

A fuzzy PID controller takes the classical PID controller as the foundation which uses the fuzzy reasoning and variable universe of discourse to regulate the PID gains ${ }^{[17]}$. The characteristics of a fuzzy system such as robustness and adaptability can be successfully incorporated into the controlling method for better tuning of PID gains.

The term self-tuning refers to the characteristics of the controller to tune its controlling parameters on-line automatically so as to have the most suitable values of those gains result in optimization of the process output. Fuzzy adaptive PID controller works on the control rules design on the basis of theoretical and experience analysis. Therefore, it can tune the gains $K_{p}, K_{i}$ and $K_{d}$, and by adjusting the other controlling parameters and factors on-line. This makes the precision of overall control higher and hence gives a better performance than the classical PID controller.

The self-tuning fuzzy PID controller, which takes error " $e$ " and rate of change-in-error " $e c$ " as the input to the controller makes use of the fuzzy controller rules to modify PID gains on-line. The self-tuning the PID controller refers to finding the fuzzy relationship between the three gains of PID, $K_{p}, K_{i}$ and $K_{d}$, and " $e$ " and "ec". According to the principle of fuzzy control, modifying the three gains is important to meet different requirements for control gains when " $e$ " and "ec" are different and making the control object produce a good dynamic and static performance. For selecting the inputs language variables of " $e$ " and " $e c$ ", and the outputs language variables of $\Delta K_{p}, \Delta K_{i}$ and $\Delta K_{d}$, choose seven fuzzy values (NB, NM, NS, ZO, PS, PM, PB) which NB denotes Negative Big, NM denotes Negative Medium, Negative Small (NS), Zero (ZO), Positive Small (PS), Positive Medium (PM) and PB denotes Positive Big.

Figure 4 shows the block diagram of a fuzzy adaptive PID controller. As can be seen from the block diagram, the fuzzy system takes two inputs ( $e$ and $e c$ ) and gives three outputs $\left(\Delta K_{p}\right.$, $\Delta K_{i}$ and $\Delta K_{d}$ ). This block diagram just shows the control of one degree of freedom of a quadrotor and we are used three other controllers like this controller for attitude and altitude control of quadrotor.

All input and output membership functions are of the same type, that is, Gauss function. Figure 5 shows these membership functions. The width of the fuzzy sets used for controllers is not the same and they have been determined by trial and error experience. The width of the fuzzy sets for output $\Delta K_{p}$ have been chosen [-60 60], $\Delta K_{i}$ have been chosen [-0.2 0.2$]$ and for $\Delta K_{d}$, $[-1010]$. And for inputs, the range for the error ' $e$ ' and error rate ' $e c$ ' have been chosen [-10 10].

The set of linguistic rules is the essential part of a fuzzy controller. As a rule of thumb, the parameters $K_{p}, K_{i}$ and $K_{d}$ are self-adjusted under different $e$ and $e c$ according to the following adjustment principles ${ }^{[18][19]}$ :

(1) When the error value $e$ is large and the error rate $e c$ is small, in order to improve the reaction speed of the system, $K_{p}$ should be larger; in order to prevent overshoot from being too large, $K_{i}$ should be smaller; and $K_{d}$ should be smaller.

(2) When the error value $e$ and the error change rate $e c$ are medium, $K_{p}$ and $K_{i}$ should be moderate and $K_{d}$ should be large.

(3) When the error value $e$ is small, $K_{p}$ should be larger. In order to reduce static error, the value of $K_{i}$ should be increased, while $K_{d}$ will change with the change rate of error.

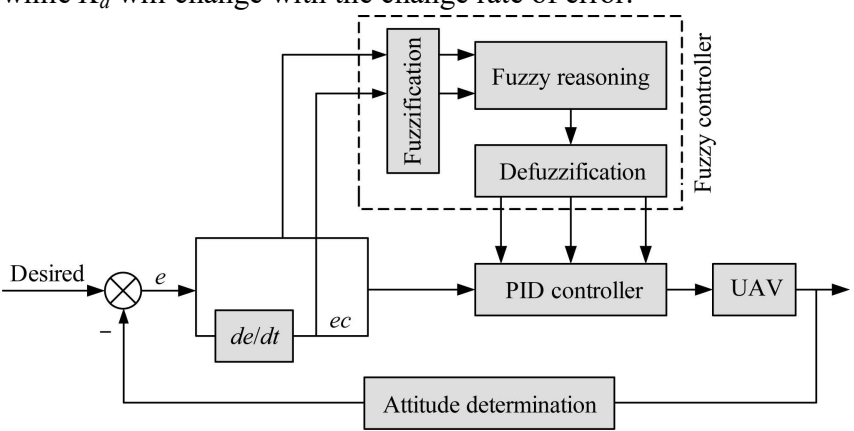

Figure 4 Block diagram of the fuzzy adaptive PID controller

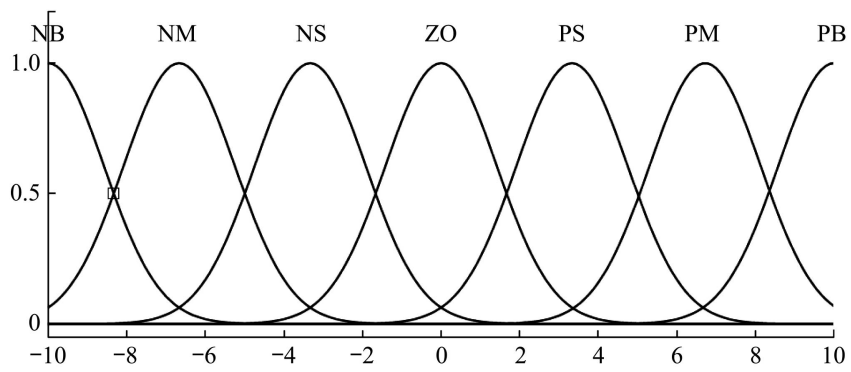

Figure 5 Membership functions for all inputs and outputs

Fuzzy adaptive PID is based on the initial PID parameters, using fuzzy logic to adjust the PID parameters intelligently, as shown in formula (14).

$$
\left\{\begin{array}{l}
K_{p}=K_{p 0}+\Delta K_{p} \\
K_{i}=K_{i 0}+\Delta K_{i} \\
K_{d}=K_{d 0}+\Delta K_{d}
\end{array}\right.
$$

The fuzzy rules for tuning the PID gains can be obtained from the above PID regulation rules and expert experience, as shown in Table 2.

The rules which were presented at the table can be read as follows: If the error is NB and the error rate is PS THEN $\Delta K_{p}$ is NS and $\Delta K_{i}$ is $\mathrm{ZO}$ and $\Delta K_{d}$ is $\mathrm{NB}$.

The output of the fuzzy system logic is fuzzy. We cannot provide these fuzzy outputs to a dynamical system as control inputs directly. The defuzzification process is needed to convert these fuzzy outputs to numbers that can represent the fuzzy output. The defuzzification should be logical, should have a high membership degree and it should correspond to the approximately middle of the graph. For the reason discussed above, we have selected a centroid defuzzifier for the controllers.

\section{Simulation and results}

In this study, we used the control variable method to study them. For example, when the flight altitude control is studied, the initial and expected values of three attitude angles are set to zero, and then the parameters of the flight altitude PID controller are manually adjusted to achieve the purpose of controlling the flight 
Table 2 Fuzzy rules for $\Delta K_{p}, \Delta K_{i}, \Delta K_{d}$

\begin{tabular}{|c|c|c|c|c|c|c|c|}
\hline \multirow{2}{*}{ Error $(e)$} & \multicolumn{7}{|c|}{ Error change (ec) } \\
\hline & NB & NM & NS & $\mathrm{ZO}$ & PS & PM & PB \\
\hline NB & $\mathrm{NB} / \mathrm{ZO} / \mathrm{PS}$ & $\mathrm{NB} / \mathrm{ZO} / \mathrm{NS}$ & $\mathrm{NB} / \mathrm{ZO} / \mathrm{NB}$ & $\mathrm{NM} / \mathrm{ZO} / \mathrm{NB}$ & $\mathrm{NS} / \mathrm{ZO} / \mathrm{NB}$ & $\mathrm{NS} / \mathrm{ZO} / \mathrm{NM}$ & $\mathrm{ZO} / \mathrm{ZO} / \mathrm{PS}$ \\
\hline $\mathrm{NM}$ & NB/NM/PS & $\mathrm{NB} / \mathrm{NM} / \mathrm{NS}$ & $\mathrm{NM} / \mathrm{NS} / \mathrm{NB}$ & NS/NS/NM & $\mathrm{NS} / \mathrm{NS} / \mathrm{NM}$ & $\mathrm{ZO} / \mathrm{ZO} / \mathrm{NS}$ & $\mathrm{PS} / \mathrm{ZO} / \mathrm{ZO}$ \\
\hline NS & $\mathrm{NM} / \mathrm{NB} / \mathrm{ZO}$ & $\mathrm{NM} / \mathrm{NM} / \mathrm{NS}$ & $\mathrm{NM} / \mathrm{NS} / \mathrm{NM}$ & NS/NS/NM & $\mathrm{ZO} / \mathrm{ZO} / \mathrm{NS}$ & $\mathrm{PS} / \mathrm{PS} / \mathrm{NS}$ & $\mathrm{PS} / \mathrm{PS} / \mathrm{ZO}$ \\
\hline $\mathrm{ZO}$ & $\mathrm{NM} / \mathrm{NB} / \mathrm{ZO}$ & $\mathrm{NM} / \mathrm{NM} / \mathrm{NS}$ & NS/NS/NS & $\mathrm{ZO} / \mathrm{ZO} / \mathrm{NS}$ & PS/PS/NS & $\mathrm{PM} / \mathrm{PM} / \mathrm{NS}$ & $\mathrm{PM} / \mathrm{PB} / \mathrm{ZO}$ \\
\hline PS & $\mathrm{NS} / \mathrm{NS} / \mathrm{ZO}$ & $\mathrm{NS} / \mathrm{NS} / \mathrm{ZO}$ & $\mathrm{ZO} / \mathrm{ZO} / \mathrm{ZO}$ & PS/PS/ZO & PS/PS/ZO & $\mathrm{PM} / \mathrm{PM} / \mathrm{ZO}$ & $\mathrm{PM} / \mathrm{PB} / \mathrm{ZO}$ \\
\hline $\mathrm{PM}$ & $\mathrm{NS} / \mathrm{ZO} / \mathrm{PB}$ & $\mathrm{ZO} / \mathrm{ZO} / \mathrm{NS}$ & $\mathrm{PS} / \mathrm{PS} / \mathrm{PS}$ & PM/PS/PS & NM/PS/PS & $\mathrm{PM} / \mathrm{PM} / \mathrm{PS}$ & $\mathrm{PB} / \mathrm{PM} / \mathrm{PB}$ \\
\hline $\mathrm{PB}$ & $\mathrm{ZO} / \mathrm{ZO} / \mathrm{PB}$ & $\mathrm{PS} / \mathrm{ZO} / \mathrm{PM}$ & $\mathrm{PM} / \mathrm{ZO} / \mathrm{PM}$ & $\mathrm{PM} / \mathrm{ZO} / \mathrm{PM}$ & $\mathrm{PM} / \mathrm{ZO} / \mathrm{PS}$ & $\mathrm{PB} / \mathrm{ZO} / \mathrm{PS}$ & $\mathrm{PB} / \mathrm{ZO} / \mathrm{PB}$ \\
\hline
\end{tabular}

altitude. When the control of pitch angle is studied, the initial and expected values of the other two attitude angles and heights are set to zero, and then the parameters of the PID controller in pitch channel are manually adjusted to control the pitch angle of aircraft. Similar processing is also used when studying the other two attitude channels. Both of the controllers were run on these two scenarios and the results are compared.

For adjusting classical PID controller gains, the trial-and-error method was used. After repeated experiments, we have set up a set of initial PID gains with good effect, among which $K_{p 0}=6$, $K_{i 0}=0.1, K_{d 0}=1.6$. By using a fuzzy system to adjust the PID gain, the PID gain will automatically change according to the fuzzy rules. You can see the results of this change in Figures 6, 7 and 8.

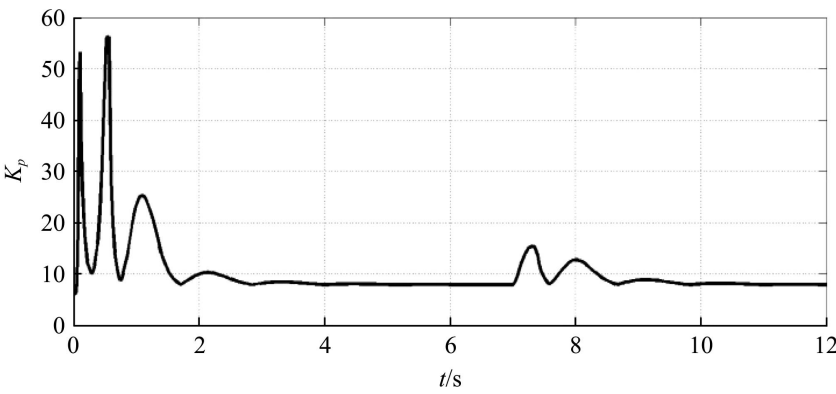

Figure 6 Fuzzy PID parameter $K_{p}$ adaptive adjustment curve

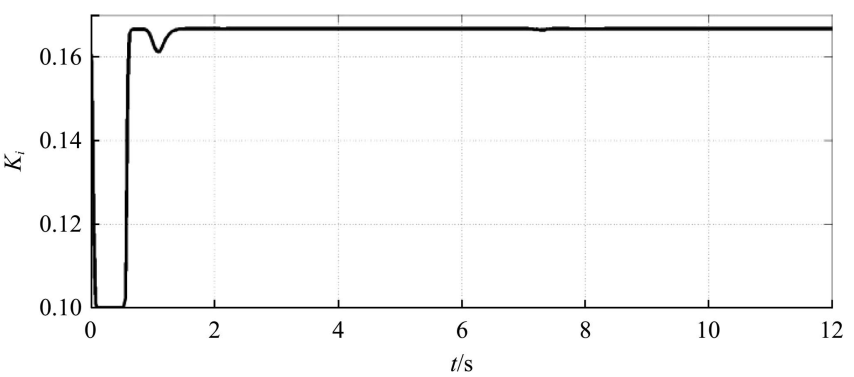

Figure 7 Fuzzy adaptive PID parameter $K_{i}$ adaptive adjustment curve

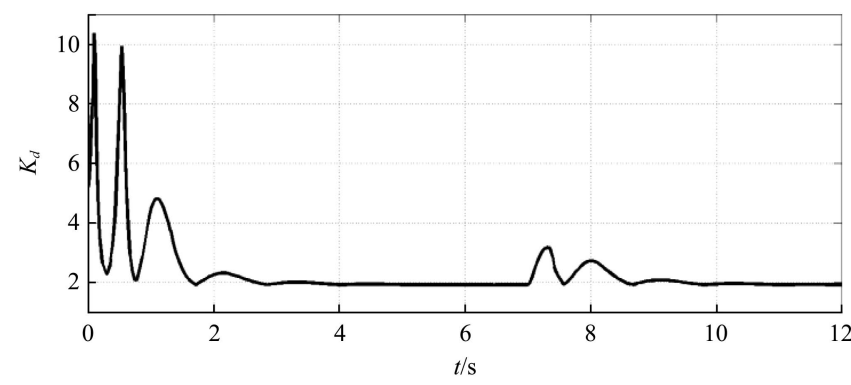

Figure 8 Fuzzy adaptive PID parameter $K_{d}$ adaptive adjustment curve

Because the simulation process of the three attitude channels is similar, only the simulation results of the pitch channel are shown below. The results of the two methods for the pitch channel were shown in Figure 9. In this figure, it showed the different responses of the pitch angle between two methods, classical PID controller and fuzzy PID controller. It is evident from this chart that the performances improved by using the fuzzy system for tuning the PID gains. Although the rise time and the steady precision of the two control methods are almost the same, the overshoot of the fuzzy adaptive PID controller is nearly $40 \%$ less than that of the classical PID controller and the setting time of the fuzzy PID controller is only half of that of the classical PID controller. In addition, in order to verify the resistance against external interference and disturbances ability of the two control algorithms, when the simulation time is 7 seconds, a pulse signal with a pulse span of $3 \%$, a period of 10 seconds and an amplitude of 10 is added. From Figure 10, we can see clearly that the resistance against external interference and disturbances ability of the fuzzy adaptive PID controller is better than that of the classical PID controller.

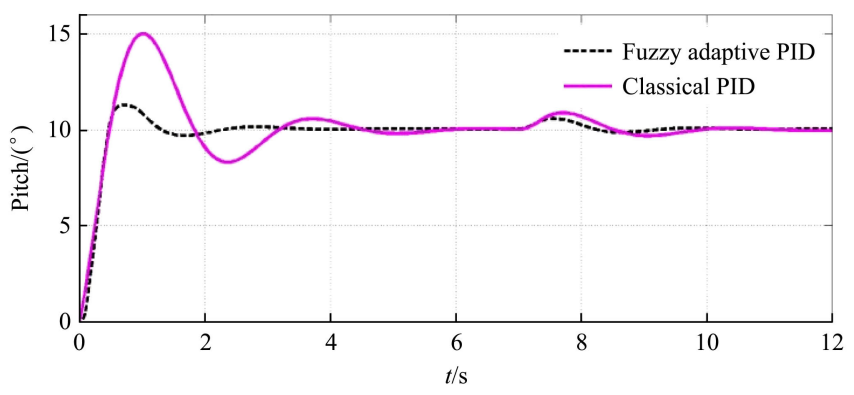

Figure 9 Comparison of simulation results between classical PID and fuzzy adaptive PID controller in pitch loop

In addition, the output of the fuzzy adaptive PID controller is only $25 \%$ of the classical PID controller, as shown in Figure 10.

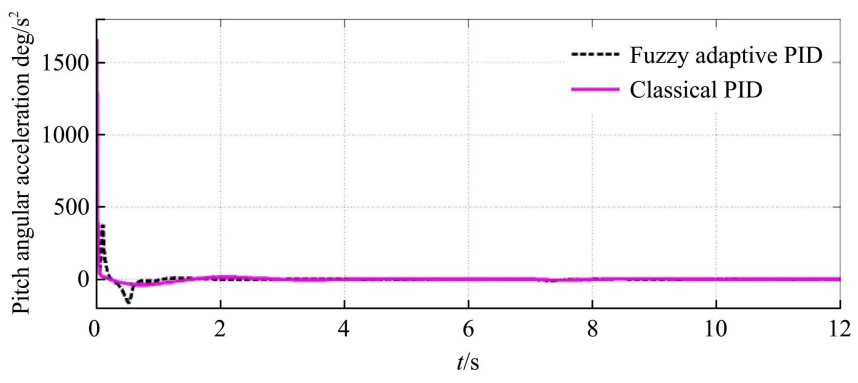

Figure 10 Simulation comparison of output of fuzzy adaptive PID and classical PID controller

The simulation effect of the altitude loop using the fuzzy adaptive PID control method is also very good, which can be seen from Figure 11. The settling time of fuzzy adaptive PID is less than $25 \%$ of classical PID. Fuzzy adaptive PID almost has no overshoot. At the same time, the anti-interference ability of fuzzy adaptive PID is also very good. 


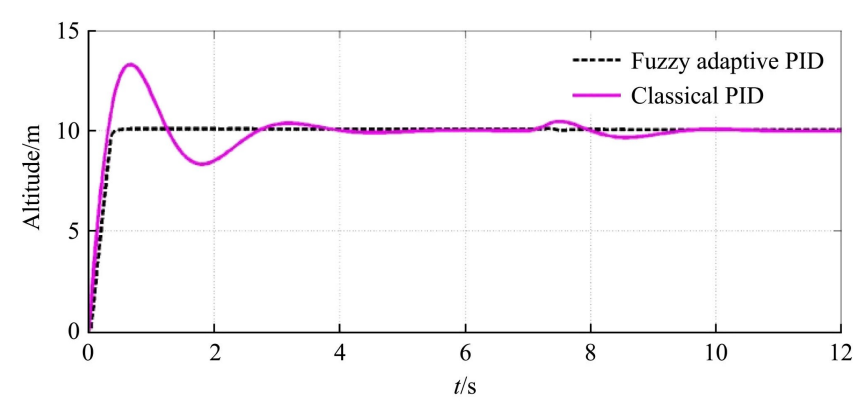

Figure 11 Comparison of simulation results between classical PID and fuzzy adaptive PID controller in altitude loop

\section{Conclusions}

In order to restrain the influence of structural parameters change and internal and external disturbances on attitude control performance and improve the adaptability of plant protection UAV, a fuzzy adaptive PID controller is designed in this study. On the basis of classical PID control, the fuzzy logic control is introduced to optimize the PID parameters. The experimental results show that the proposed method can effectively realize the attitude control of plant protection UAV, and has good static and dynamic control performance and good adaptability. The fuzzy adaptive PID control method proposed in this study can solve some problems existing in classical PID control. It has good application prospects in attitude control of plant protection UAV.

\section{Appendix}

Some constants which may be used in the simulation of the quadrotor are shown in Table A.

Table A Constants List

\begin{tabular}{clc}
\hline Symbol & \multicolumn{1}{c}{ Description and unit } & Value \\
\hline$m$ & Mass of the quadrotor $(\mathrm{kg})$ & 1.25 \\
$l$ & Center of quadrotor to the center of propeller distance $(\mathrm{m})$ & 0.2 \\
$I x$ & Body moment of inertia around the x-axis $\left(\mathrm{Nms}^{2}\right)$ & 0.0024 \\
$I y$ & Body moment of inertia around the y-axis $\left(\mathrm{Nms}^{2}\right)$ & 0.0025 \\
$I z$ & Body moment of inertia around the $\mathrm{z}$-axis $\left(\mathrm{Nms}^{2}\right)$ & 0.0047 \\
$b$ & Thrust factor & $2.92 \times 10^{-6}$ \\
$d$ & Drag factor & $1.12 \times 10^{-7}$ \\
\hline
\end{tabular}

\section{Acknowledgements}

The authors would like to thank their colleagues for their support of this work. This research work was supported by Project of Scientific Operating Expenses from Ministry of Education of China (2017PT19) and National Natural Science Foundation of China (31761133019).

\section{[References]}

[1] He X K, Bonds J, Herbst A, Langenakens J. Recent development of unmanned aerial vehicle for plant protection in East Asia. Int $\mathrm{J}$ Agric \& Biol Eng, 2017; 10(3): 18-30.

[2] Huang X. Design and implementation of plant-protection UAV based on STM32F427. Diss. Southwest University of Science and Technology, 2016.

[3] Liu C, Zhang C L, Wang S W, Wang R T, Zhang L Y, Lv T, et al. Longitudinal attitude control system design and simulation of agricultural UAV. Agricultural Mechanization Research, 2016; 10: 6-10.

[4] Qiu D. The implementation of flight control unit for agricultural multi-rotors UAV. Diss. Xidian University, 2017.

[5] Huang Y, Zhang W G. Development of active disturbance rejection controller. Control Theory \& Applications, 2002; 19(4): 485-492.

[6] Han J Q. From PID to active disturbance rejection control. IEEE Transactions on Industrial Electronics, 2009; 56(3): 900-906.

[7] Gao H, Liu C, Guo D, Liu J. Fuzzy adaptive PD control for quadrotor helicopter. IEEE International Conference on Cyber Technology in Automation, 2015.

[8] Muhyiddin Y A. Comparative study of conventional PID and fuzzy-PID for DC motor speed control. Masters thesis. Universiti Tun Hussein Onn Malaysia, 2013.

[9] Sim S Y, Goh H H, Utomo W M. A comparative study of conventional PID and intelligent Fuzzy-PID Ford DC motor speed control. Journal of Fundamental and Applied Sciences, 2018; 10(5S): 282-297.

[10] Dierks T, Jagannathan S. Output feedback control of a quadrotor UAV using neural networks. IEEE Transactions on Neural Networks, 2010; 21(1): 50-66.

[11] Liu H, Bai Y, Lu G, Shi Z, Zhong Y. Robust tracking control of a quadrotor helicopter. Journal of Intelligent \& Robotic Systems, 2014; 75(3-4): 595-608.

[12] Li L. Dynamic analysis and PID control for a quadrotor. International Conference on Mechatronics \& Automation, IEEE, 2011.

[13] Salih A L, Moghavvemi M, Mohamed H A F, Gaeid K S. Modelling and PID controller design for a quadrotor unmanned air vehicle. International Conference on Automation, IEEE Computer Society, 2010.

[14] Azzam A, Wang X W. Quad rotor arial robot dynamic modeling and configuration stabilization. International Asia Conference on Informatics in Control, IEEE, 2010.

[15] Gao H, Liu C, Guo D, Liu J. Fuzzy adaptive PD control for quadrotor helicopter. IEEE International Conference on Cyber Technology in Automation, IEEE, 2015.

[16] Shubhangi R, Hemant A. Comparative analysis among fuzzy PI controller, fuzzy PID controller and fuzz logic controller for the speed control of induction motor. International Journal of Enhanced Research in Science Technology \& Engineering, 2013; 2(8): 1-7.

[17] Wahid N, Hassan N. Self-tuning fuzzy PID controller design for aircraft pitch control. 2012 Third International Conference on Intelligent Systems Modelling and Simulation, Kota Kinabalu, 2012; pp.19-24.

[18] Yener T. Fuzzy PID controller for propeller pendulum. Istanbul University: Journal of Electrical and Electronics Engineering, 2017; 17(1): 3175-3180.

[19] Arbab N K, Dai Y P, Syed A A, Xu X Y. Stable hovering flight for a small unmanned helicopter using fuzzy control. Mathematical Problems in Engineering, 2014; Article ID 208253, 17p. doi: 10.1155/2014/208253 